\title{
LA CONJUNCIÓN DEL DERECHO Y LA CIENCIA PARA LA DETERMINACIÓN DE LA SOSTENIBILIDAD DE LAS ENERGÍAS RENOVABLES EN EL FUTURO PARADIGMA ENERGÉTICO DE EUROPA
}

\author{
THE CONFLUENCE BETWEEN LAW AND SCIENCE IN DETERMINING THE \\ SUSTAINABILITY OF RENEWABLE ENERGIES IN THE FUTURE ENERGY \\ PARADIGM OF EUROPE
}

\author{
MARÍA PASCUAL NúÑEZ \\ Investigadora Predoctoral \\ Centro Internacional de Estudios de Derecho Ambiental (CIEDA-CIEMAT) \\ Maria.Pascual@cieda.es \\ CARLOS SANZ SAN MigUEL \\ Investigador Predoctoral \\ Centro de Desarrollo de Energías Renovables (CEDER-CIEMAT) \\ carlos.sanzsanmiguel@ciemat.es
}

Fecha de recepción: 24/12/2020 - Fecha de aceptación: 27/03/2021

RESUMEN: Una de las reticencias sociales más extendidas acerca del uso de combustibles de biomasa son las emisiones que producen. En el caso de la biomasa forestal, el recuento y la gestión del carbono absorbido, los posibles daños a la biodiversidad y a la calidad de los aprovechamientos forestales, y el posible aumento de emisiones son puntos conflictivos para el sector ambientalista. Por el contrario, el sector técnico apuesta por una gestión forestal sostenible, mediante el uso de técnicas avanzadas que permitan conocer cómo funcionan los mecanismos de los bosques como sumideros de carbono.

Los riesgos ambientales del uso energético de los biocombustibles pueden ser revertidos mediante el empleo de parámetros técnicos. La normativa europea establece criterios de sostenibilidad para la biomasa con destino energético, pero no fija parámetros acerca de la sostenibilidad del resto de fuentes renovables.

Este trabajo se basa en la necesaria imbricación entre la técnica normativa consagrada el programa europeo "Legislar mejor para obtener mejores resultados - Un programa de la UE" y la ciencia, por una mayor sostenibilidad del uso de fuentes de energía renovables.

Palabras clave: Análisis de ciclo de vida, bioenergía, biocombustibles, criterios de sostenibilidad, economía circular, energías renovables, gestión forestal sostenible, Plan Nacional Integrado de Energía y Clima.

ABSTRACT: One of the most widespread social reservations about the use of biomass fuels is the emissions they produce. In the case of forest biomass, the counting and management of the absorbed carbon, the potential harm to biodiversity and the quality of forest harvesting, and the probable rise in emissions are points of conflict for the environmental sector. On the other hand, the technical sector is committed to sustainable forest management, through the use of advanced techniques that allow us to understand how the mechanisms of forests work as carbon sinks.

The environmental risks of the energy use of biofuels can be reversed through the use of technical parameters. European regulations establish sustainability criteria for biomass for energy purposes, but do not set parameters for

${ }^{1}$ COMISIÓN EUROPEA. Comunicación "Legislar mejor para obtener mejores resultados - Un programa de la UE" [COM (2015) 215 final]. 
La conjunción del derecho y la ciencia para la determinación de la sostenibilidad de las energías renovables en

el futuro paradigma energético de Europa

the sustainability of other renewable sources.

This work is based on the necessary imbrication between the regulatory technique enshrined in the European programme "Better Regulation for better results - An EU agenda" and science, for greater sustainability in the use of renewable energy sources.

Keywords: Life cycle analysis, bioenergy, biofuels, sustainability criteria, circular economy, renewable energies, sustainable forest management, Integrated National Energy and Climate Plan.

SUMARIO: I. INTRODUCCIÓN Y METODOLOGÍA. II. EL PNIEC COMO ELEMENTO INTEGRADOR DE LA PLANIFICACIÓN SECTORIAL. El CASO DE LA SILVICULTURA. III. LOS CRITERIOS DE SOSTENIBILIDAD DEL USO ENERGÉTICO DE LA BIOMASA. 1. Condicionantes ambientales de la energía de biomasa: un puzle de parámetros técnicos. 1.1. El principio de proximidad del recurso a la instalación como vector de sostenibilidad y de reducción de emisiones de GEI. 1.2. La biomasa es una energía renovable gestionable. 1.3. Gestión Forestal Sostenible. 1.4. La biomasa forestal circular y los biocarburantes avanzados. 1.5. La importancia de las metodologías de contabilización de GEI y de los sistemas de modelización. IV. RETICENCIAS AL USO ENERGÉTICO DE LA BIOMASA FORESTAL RESIDUAL. V. EL AUTOCONSUMO COMO ELEMENTO PIVOTE DE LA GENERACIÓN DISTRIBUIDA EN EL NUEVO MODELO A BASE DE ENERGÍAS RENOVABLES. VI. RECAPITULACIÓN Y CONCLUSIONES. VII. REFERENCIAS BIBLIOGRÁFICAS. 


\section{INTRODUCCIÓN Y METODOLOGÍA}

Las políticas en materia de clima y energía para el periodo 2021-2030 deben concretarse en los planes nacionales de clima y energía (PNIEC), instrumentos concebidos por el Reglamento 2018/1999 del Parlamento Europeo y del Consejo, de 11 de diciembre de 2018, sobre la gobernanza de la Unión de la Energía y de la Acción por el Clima, de aplicación directa en los Estados miembros. Estos planes se estructuran en torno a cinco dimensiones: descarbonización y energías renovables, eficiencia energética, seguridad energética, mercado interior de la energía e investigación, innovación y competitividad, y establecen la "hoja de ruta" hacia el cumplimiento de los objetivos previstos en la normativa comunitaria.

Para la dimensión sobre descarbonización de la economía y la internación de las fuentes renovables, deberá integrar las prerrogativas de la Directiva 2018/2001 del Parlamento Europeo y del Consejo, de 11 de diciembre de $2018^{2}$ (DFERII en lo que sigue), relativa al fomento del uso de energía procedente de fuentes renovables; del Reglamento 2018/841 del Parlamento Europeo y del Consejo, de 30 de mayo de 2018, sobre la inclusión de las emisiones y absorciones de gases de efecto invernadero resultantes del uso de la tierra, el cambio de uso de la tierra y la silvicultura en el marco de actuación en materia de clima y energía hasta 2030 (Reglamento UTCUTS), y del Reglamento 2018/842 del Parlamento Europeo y del Consejo, de 30 de mayo de 2018, sobre reducciones anuales vinculantes de las emisiones de gases de efecto invernadero por parte de los Estados miembros entre 2021 y 2030, que contribuyan a la acción por el clima, con objeto de cumplir los compromisos contraídos en el marco del Acuerdo de París. En concreto, el artículo 5 del citado Acuerdo alude a la mejora y conservación de los depósitos y sumideros de carbono, mencionando explícitamente al sector forestal. La traslación de estos compromisos al ámbito comunitario condiciona ineludiblemente el uso energético de la biomasa, como se estudiará más adelante.

En el caso de la dimensión de la eficiencia energética, debe hacerse lo correspondiente con las disposiciones de la Directiva 2012/27/UE del Parlamento Europeo y del Consejo, de 25 de octubre de 2012, relativa a la eficiencia energética.

Finalmente, deberán alcanzarse los objetivos previstos para las dimensiones de la seguridad energética, el mercado interior de la energía y de la investigación, innovación y competitividad.

Dicho lo anterior, la planificación en materia de energía y clima es como una tela de araña. Un entramado de medidas y líneas de actuación que articulan una red que se extiende a través de distintos ámbitos sectoriales (transportes, infraestructuras, urbanístico, hidrológico, forestal, adaptación al cambio climático, calidad de aire, residuos, biodiversidad, ...), y que debe considerar las competencias que ostentan las entidades territoriales a nivel internacional, nacional, autonómico y local.

La Gobernanza comunitaria, cuya acción se cataliza a través de la Unión de la Energía, implica la participación de Estados miembros con distintas capacidades, cuestión que se tiene en cuenta en la fórmula del Anexo II para prorratear los objetivos comunitarios. Si bien, se trata de una fórmula indicativa, y los objetivos derivados de su aplicación pueden ser ampliados a discreción de los Estados miembros en su planificación, como ha ocurrido en el caso de España. El PNIEC español prevé una serie de medidas cuya aplicación eficaz podrá traducirse en: i) un $23 \%$ de reducción de GEI respecto a 1990; ii) $42 \%$ de renovables sobre el uso final de la energía; y iii) $39,5 \%$ de mejora de la eficiencia energética. Asimismo, destaca el objetivo

\footnotetext{
2. Directiva 2018/2001, del Parlamento Europeo y del Consejo, de 11 de diciembre de 2018, relativa al fomento del uso de energía procedente de fuentes renovables [DO L 328 de 21.12.2018, p. 82/209].
} 
sectorial de que el $74 \%$ de la generación eléctrica sea de origen renovable.

\section{EL PNIEC COMO ELEMENTO INTEGRADOR DE LA PLANIFICACIÓN SECTORIAL. EL CASO DE LA SILVICULTURA}

El PNIEC español constituye el marco al que deben adecuarse el resto de la planificación sectorial (transportes, infraestructuras, urbanística, hidrológica, forestal, de adaptación al cambio climático, de calidad de aire, residuos, biodiversidad, forestal, etcétera) y los proyectos que tengan incidencia en el medio ambiente. Del análisis de las medidas previstas en el PNIEC, se deduce una reducción del 39\% en las emisiones de gases de efecto invernadero, 13 puntos por encima de lo asignado en el Anexo I del Reglamento 2018/842, y se estima que gracias al sector UTCUTS se podrán eliminar cerca de $1 \mathrm{Mt}$ eq. de $\mathrm{CO}_{2}$, especialmente en zonas forestales. En conexión con el sector de la silvicultura, su medida 1.11 prevé la promoción de las energías procedentes de biomasa con criterios de sostenibilidad y el apoyo económico para el aprovechamiento de la biomasa, o la penalización del depósito de residuos en vertedero.

La incidencia de esta planificación en el medio ambiente justifica la sujeción del PNIEC al trámite de evaluación ambiental ordinaria, como pone de manifiesto el artículo 6 de la Ley 21/2013, de 9 de diciembre, de evaluación ambiental (LEA) ${ }^{3}$. No obstante, el despliegue de las acciones y medidas contenidas en el PNIEC requiere de actuaciones complementarias que se ejecutarán a través de planes y proyectos concretos, que no quedan exentos de su evaluación ambiental individualizada. En este sentido, la evaluación ambiental estratégica (EAE) del PNIEC $^{4}$ propone medidas y recomendaciones para la transformación sectorial, que afectan al ámbito forestal y al impulso de los biocombustibles.

\section{LOS CRITERIOS DE SOSTENIBILIDAD DEL USO ENERGÉTICO DE LA BIOMASA}

La sostenibilidad de la biomasa, especialmente la de origen silvícola, ha sido recientemente objeto de crítica por una serie de trabajos periodísticos, enmarcados en la campaña "Money to burn". La polémica ha surgido durante la consulta pública destinada a la revisión del marco jurídico en materia de energía en el seno de la Comisión Europea, siendo una de las cuestiones tratadas la posibilidad de modificar los criterios de sostenibilidad de la bioenergía en general y de la forestal en particular.

La Directiva 2018/2001 prevé en su artículo 29 la última versión de los criterios de sostenibilidad, y de reducción de las emisiones de gases de efecto invernadero, para los

\footnotetext{
${ }^{3}$ El sometimiento del PNIEC a evaluación ambiental estratégica (EAE) no exime a los planes y proyectos individualizados, amparados por el mismo, de su sometimiento al trámite de evaluación de impacto ambiental. El nuevo marco para el impulso de las energías renovables exige simplificar la tramitación administrativa de los nuevos proyectos de energías renovables, no obstante, la EAE prevé que esta simplificación no perjudique a la evaluación ambiental. Sobre la integración de la Evaluación de Impacto Ambiental de proyectos en el nuevo marco de simplificación administrativa para el impulso de las fuentes renovables, BELTRÁN CASTELLANOS, J. M., "Instalaciones de energías renovables. El reto de la unificación y simplificación de los procedimientos" en VALENCIA MARTÍN, G. y ROSA MORENO, J., La transformación renovable del modelo energético. Thomson Reuters - Aranzadi, 2020, pp. 383-426.

${ }^{4}$ Publicada mediante la Resolución de 30 de diciembre de 2020, de la Dirección General de Calidad y Evaluación Ambiental, por la que se formula la declaración ambiental estratégica del Plan Nacional Integrado de Energía y Clima 2021-2030 [BOE-A-2021-421].

${ }^{5}$ RICO, J., "La posible modificación de los criterios de sostenibilidad de la bioenergía desata el debate en Europa". Energías Renovables, 21 de enero de 2021. Disponible en https://www.energias-renovables.com/biomasa/la-posiblemodificacion-de-los-criterios-de-20210121.
} 
biocarburantes, biolíquidos y combustibles de biomasa, cuya verificación es exigida por imperativo del artículo 30. Seguidamente, el artículo 31 dispone cómo debe calcularse el efecto de los biocarburantes, biolíquidos y combustibles de biomasa en las emisiones de GEI, conforme a las determinaciones de los Anexos V y VI.

Las emisiones de gases de efecto invernadero constituyen un problema inherente a la producción de energía renovable de cualquier fuente. Si bien la energía de la biomasa requiere de la explotación de recursos de origen biológico, fuentes como la fotovoltaica o la eólica precisan de importantes cantidades de recursos minerales y otros materiales, cuya extracción, procesado y transporte también generan un importante impacto ambiental, por lo que es necesario establecer una frontera clara entre energía procedente de fuentes renovables y energía limpia. La normativa de fomento de energías renovables se centra, sin embargo, en establecer prerrogativas para el uso energético sostenible de los productos de origen biológico.

Los criterios de sostenibilidad surgen por la necesidad de integrar las energías renovables principalmente en los sectores de la energía y del transporte, de tal manera que se produzcan biocombustibles conforme a unos parámetros que garanticen una reducción efectiva del impacto ambiental con respecto a los combustibles fósiles. Son obligatorios en determinados tipos de instalaciones, en concreto, aquellas que produzcan electricidad, calefacción y refrigeración, y para la producción de combustibles sólidos y gaseosos, de una determinada potencia térmica nominal ${ }^{6}$. Los nuevos criterios de sostenibilidad incorporan una remisión explícita al artículo 5 del Acuerdo de París, cuya finalidad es equilibrar los gases emitidos y los que pueden ser absorbidos a partir de 2050, es decir, la consecución del objetivo de "cero emisiones netas". La capacidad de absorción de $\mathrm{CO}_{2}$ de la materia biológica es una preocupación a la que debe atenderse si se quiere considerar esta fuente como sostenible.

El entramado de criterios técnicos que requiere el uso sostenible de la biomasa puede generar controversias dado que la determinación de parámetros ambientales para la biodiversidad, la protección de los suelos o las emisiones de gases durante la combustión, transporte y recolección del recurso es una materia en constante evolución, asociadas a mejoras en equipos de medición y normalización de métodos de ensayo. Sin embargo, no deben obviarse otros resultados científicos igualmente válidos, como pueden ser los beneficios relativos al aumento de la absorción de carbono o de la superficie boscosa, derivadas de una valorización apropiada del producto, una gestión sostenible, y una explotación energética tecnológicamente desarrollada.

De la misma manera, otras fuentes renovables, resultan beneficiadas en la comparación, al ser el criterio decisivo las emisiones de gases de efecto invernadero sólo en la fase de generación de energía. Si aplicamos el análisis de ciclo de vida a todo el proceso, es inevitable cuestionar, entre otros, qué ocurre con el agotamiento de los minerales necesarios para el despliegue de la infraestructura que la ansiada transición energética renovable requiere. Intentaremos dar respuesta a este interrogante más adelante.

\section{Condicionantes ambientales de la energía de biomasa: un puzle de parámetros técnicos}

Volviendo a la energía de la biomasa, la Unión Europea ha asumido el paradigma del artículo 5 del Acuerdo de París mediante el Reglamento 2018/841, cuya finalidad es alcanzar el

\footnotetext{
${ }^{6}$ Artículo 29.1 y 11 de la DFERII. El apartado 1 vincula a las instalaciones de producción de energía eléctrica, calefacción y refrigeración, así como a las de producción de los combustibles sólidos con una potencia térmica nominal igual o superior a $20 \mathrm{MW}$ y combustibles gaseosos con una potencia térmica nominal igual o superior a 2MW. El apartado 11 concreta las especificaciones aplicables a las instalaciones de producción de electricidad.
} 
objetivo de la "norma de deuda cero". Con esta regulación se pretende optimizar la capacidad de absorción de los suelos desde una perspectiva productivista de los mismos. Los criterios de sostenibilidad del precitado artículo 29 aluden a la toma en consideración de los usos de la tierra donde se producen las materias primas para la producción de biocombustibles.

La incertidumbre sobre la sostenibilidad de los usos energéticos de la biomasa se deduce de la propia DFERII, que alude en su Anexo V a la revisión en 2020 de las directrices para calcular las reservas de carbono en suelo, basándose en las Directrices de 2006 del IPCC para los inventarios nacionales de GEI, volumen 4, y de acuerdo con el Reglamento 525/2013 y con el Reglamento 2018/841, sobre las normas para calcular el impacto de los biocarburantes, biolíquidos y sus combustibles fósiles de referencia en las emisiones de gases de efecto invernadero.

En el contexto de revisión de la DFERII, el Centro Común de Investigación (CCI) de la Comisión Europea ha publicado recientemente un informe que orienta la eventual modificación de los criterios de sostenibilidad de la bioenergía de origen forestal ${ }^{7}$. Se trata de un informe enmarcado en la Estrategia de Biodiversidad para $2030^{8}$, que reconoce la complejidad de analizar la sostenibilidad de la biomasa leñosa, para cuya determinación no existe una respuesta única. A grandes rasgos, establecer el impacto de la bioenergía debe conjugar parámetros para la reducción de emisiones de gases de efecto invernadero y criterios referidos a los usos del suelo y a la biodiversidad, cuya aplicación se sugiere extender a todos los productos forestales y no sólo a la energía?

Para atender, coordinar y mejorar este entramado de requisitos ambientales, es importante mejorar la calidad y la disponibilidad de datos sobre el sector forestal y el uso energético de la madera. Para los expertos del CCI, el problema no radica tanto en la falta de información como sí en la existencia de lagunas y en que no existe una base de datos única que facilite una imagen compleja de cómo realizar la gestión. A ello se añade que la información que proporcionan los Estados miembros no está armonizada, existiendo desfases entre la producción de bioenergía notificada y la real.

\subsection{El principio de proximidad del recurso a la instalación como vector de sostenibilidad y de reducción de emisiones de GEI}

El Anexo VI de la DFERII establece, como se ha especificado con anterioridad, las normas para calcular el impacto de los combustibles de biomasa y los combustibles fósiles de referencia en las emisiones de gases de efecto invernadero. Sin embargo, hay elementos asociados a la sostenibilidad de la biomasa como fuente energética a los que no se les presta la suficiente atención. El principio de proximidad del recurso permite reducir considerablemente las emisiones de $\mathrm{CO}_{2}$ asociadas a los procesos de producción de energía. Así, en los valores típicos y por defecto de las reducciones de gases de efecto invernadero, se incluyen distancias de transporte de más de $10.000 \mathrm{~km}$. Para hacer más eficiente la transición a una economía descarbonizada es preferible el consumo de recursos que cumplan parámetros o criterios de circularidad y proximidad, minimizando su transporte.

\footnotetext{
${ }^{7}$ CAMIA, A., GIUNTOLI, J., JONSSON, K., ROBERT, N., CAZZANIGA, N., JASINEVIČIUS, G., AVITABILE, V., GRASSI, G., BARREDO CANO, J. I. y MUBAREKA, S., The use of woody biomass for energy production in the EU. Publications Office of the European Union, Luxembourg, 2020.

${ }^{8}$ COMISIÓN EUROPEA. Estrategia de Biodiversidad para 2030 [COM (2020) 380].

9 RICO, J., "Un informe de la CE sugiere que los criterios de sostenibilidad se apliquen a todos los productos forestales, no solo a la bioenergía", Energías Renovables, 2021, 4 de febrero de 2021. Disponible en https://www.energias-renovables.com/biomasa/un-informe-de-la-ce-sugiere-que-20210204
} 
Si se piensa en la transición a un modelo energético hipocarbónico, la mera transformación o adaptación de las grandes centrales eléctricas consumidoras de combustibles fósiles en centrales de biomasa, en el sector de la generación energética, así como la sustitución de combustibles fósiles por biocarburantes en el sector del transporte, sin adaptación tecnológica o implementación de dichos parámetros, sólo reemplazará un desastre por otro ${ }^{10}$. En términos prácticos, el despliegue del principio de proximidad debería traducirse en una mayor dispersión de los centros de producción y la integración en la red de muchos puntos de consumo cercanos a la biomasa. Ello en contraposición al planteamiento actual, de adaptación de grandes centrales de producción, que requiere desplazar más materias energéticas. Asimismo, el sector de la calefacción supone un nicho de mercado sostenible cuando se emplea en redes urbanas sitas cerca de los recursos de origen biológico.

Para superar los obstáculos normativos que lastran la aplicación del principio de proximidad de origen del recurso, la EAE del PNIEC recomienda fomentar dicho principio para el uso energético de la biomasa. Desde luego, su aplicación reforzada podría traer consigo beneficios económicos, ambientales y sociales. Un ejemplo es la posibilidad de generar cadenas de valor para los productos del sector silvícola, impulsando, a las regiones donde se sitúan estos recursos. Estos recursos suelen ubicarse en zonas eminentemente rurales y su movilización desde parámetros de sostenibilidad puede contribuir a desarrollar un tejido industrial en dichas áreas, generando empleo, fijando población...11

Debe enfatizarse que, al hablar de industrialización del medio rural en términos de la generación de mercados entorno a productos biológicos, no deben perderse de vista los compromisos suscritos en el Acuerdo de París. Es imperativo salvaguardar y mejorar los sumideros de carbono, de modo que el sector agro-silvícola debería verse sometido a una profunda transformación a lo largo de la próxima década. En este sentido, la EAE del PNIEC español alienta que la Estrategia de Descarbonización a Largo Plazo refuerce la promoción de producto $\mathrm{Km} 0$.

\subsection{La biomasa es una energía renovable gestionable}

El carácter gestionable de la biomasa como fuente energética es uno de sus mayores valores. En el contexto de cambio climático, el fenómeno meteorológico Filomena ha puesto de manifiesto posibles debilidades del futuro sistema energético descarbonizado, como la ausencia de generación fotovoltaica y eólica, así como del vehículo eléctrico.

Entre los problemas que acarrean la integración y dependencia de un mayor porcentaje de generación renovable en nuestro parque eléctrico destaca el aumento de las necesidades de almacenamiento y la pérdida, por tanto, de flexibilidad ante picos en la demanda. Un ejemplo reciente ha sido el aumento de la demanda eléctrica en un 15,05\% durante la semana del 4 al 10 de enero, coincidiendo con la ola de frío. Si además pensamos en la interconexión de los distintos países de la Unión Europea, entre los que no existe demasiada variabilidad climática, es probable que un día nublado en España también lo sea en Portugal o Francia, y no se pueda satisfacer el déficit en energía fotovoltaica necesaria. No podemos olvidar que las energías solar

\footnotetext{
${ }^{10}$ MOVIMIENTO MUNDIAL POR LOS BOSQUES TROPICALES “La transformación de centrales a carbón para combustión de biomasa sólo reemplaza un desastre por otro", Boletín Mensual, núm. 189, 2013, 7 de septiembre de 2020. Disponible en https://wrm.org.uy/es/articulos-del-boletin-wrm/seccion2/la-transformacion-de-centrales-acarbon-para-combustion-de-biomasa-solo-reemplaza-un-desastre-por-otro/

${ }^{11}$ RODRÍGUEZ CHAVES-MIMBRERO, B., "Apuesta de los montes como sumideros de carbono y como fuente de riqueza en el ámbito rural”, en LOPEZ RAMÓN, F., Observatorio de Políticas Ambientales, Ed.1", Aranzadi, CIEDA - CIEMAT, 2017, pp. 455-482.
} 
y eólica dependen íntegramente de la meteorología, de modo que el uso energético de la biomasa puede constituir un factor estratégico para el sostenimiento del suministro ${ }^{12}$.

Sin embargo, la biomasa no contó con potencia asignada por tecnología en la primera subasta de energías renovables celebrada en nuestro país ${ }^{13}$. Las ofertas correspondientes debían presentarse entre los 1000 MW no asignados por tecnología. Sí que se prevé la asignación de 140 MW para la subasta de 2021, 260 MW para 2023 y 308 MW acumulados para $2025^{14}$.

Desde el punto de vista de la adaptación de las redes, entre los beneficios de la biomasa, se trata de una fuente gestionable que no acarrea los problemas de almacenamiento asociados a las fuentes fotovoltaica y eólica. Nuestra red ya dispone de sistemas de flexibilización, como puede ser el almacenamiento hidráulico (o de cualquier otro tipo), que puede ser integrado con otras fuentes de producción renovable, y la quema de combustibles fósiles en grandes centrales. Aquí es donde la biomasa puede tener un papel sustitutivo a efecto inmediato, pero se ha de integrar en proyectos de producción próxima. Por ejemplo, en una red urbana de calefacción, la biomasa es un recurso que puede estar disponible todo el año, amén de que el hecho de unificar la quema del combustible hace rentable la inversión en equipos de limpieza de humos, reduciendo en mayor medida la emisión de gases de efecto invernadero y haciendo si cabe más sostenible su uso. En el caso de la energía fotovoltaica, su disponibilidad es mayor en verano, por lo que no resulta tan atractiva.

Finalmente, el concepto de gestión en red de la producción de energía por parte de los Estados miembros debe estar vinculada al control coordinado del recurso energético, especialmente en el caso de la biomasa, dado que los espacios forestales pueden ocupar el territorio de varios países comunitarios. Resulta relevante el hecho de que la biomasa es una materia disponible en el ámbito europeo y, especialmente, en España ${ }^{15}$. Ello en contraposición a los minerales necesarios para el despliegue de la infraestructura de la energía renovable, de los cuales el mercado de la Unión Europea es deficitario y dependiente de otras potencias. El marco de la sostenibilidad de las fuentes energéticas renovables debe tomar en consideración las emisiones asociadas a la extracción, manipulación, facturación y transporte de estos minerales, como hace con las distintas fuentes de biomasa.

\subsection{Gestión Forestal Sostenible}

La gestión forestal sostenible constituye una de las piedras angulares del cumplimiento de los objetivos acordados en París en el año 2015. Se trata de un elemento complejo en el que converge la ordenación del territorio con los procedimientos voluntarios de certificación forestal. La Estrategia Forestal Española ${ }^{16}$ informa de cómo la Ordenación de Montes es el

\footnotetext{
12 "Ence ayuda a mantener el suministro de energía renovable durante el temporal Filomena", Europapress, 13 de enero de 2021. Disponible en https://www.europapress.es/economia/energia-00341/noticia-ence-ayuda-mantenersuministro-energia-renovable-temporal-filomena-20210113113442.html
}

13 Asociaciones del sector, como APPA Biomasa y Avebiom, presentaron alegaciones a la orden reguladora del mecanismo de subastas, con la pretensión de que se asignara más potencia a esta fuente y se tuviera el coste mayor de producción de energía, pero también su carácter gestionable.

${ }^{14}$ RICO, J., "Claro que podíamos presentar biomasa a la subasta, pero a un precio inasumible". Energías Renovables, 2021, 29 de enero de 2021. Disponible en https://www.energias-renovables.com/biomasa/a-claro-que-podiamospresentar-biomasa-a-20210129

${ }^{15}$ RODRÍGUEZ CHAVES-MIMBRERO, B., "Montes: la gestión sostenible en la normativa de última generación" en LOPEZ RAMÓN, F., Observatorio de Políticas Ambientales, Ed.1ª, Aranzadi, CIEDA - CIEMAT, 2015, Madrid. pp. 645-693.

${ }^{16}$ MINISTERIO DE AGRICULTURA, PESCA Y ALIMENTACIÓN, Estrategia Forestal Española. Segunda parte; Propuestas de Política Forestal, 22 de diciembre de 2020. Disponible en https://www.mapa.gob.es/es/desarrollo- 
basamento de la gestión forestal sostenible. Esta ordenación es una forma específica de planificación que obedece a proteger la multifuncionalidad de estos espacios y a la mejora de sus capacidades. En España, los instrumentos de ordenación constituyen un elemento a revisar en la auditoría prevista para la concesión de la certificación forestal, de modo que sólo cuando el terreno se gestione conforme a criterios de sostenibilidad y cuente con el instrumento correspondiente de ordenación será susceptible de certificación ${ }^{17}$.

Los mecanismos de certificación de la gestión forestal sostenible son instrumentos de mercado que se circunscriben al ámbito privado. Permiten abordar el aprovechamiento de los recursos silvícolas, ofreciendo una "hoja de ruta" acerca de las prácticas a desarrollar en los mismos, coherentes con la ordenación del aprovechamiento. Así, se facilita a sus titulares la transición hacia un modelo de gestión que obliga a que la productividad de estos espacios esté vinculada a su protección y mejora.

La preocupación de la DFERII por el uso sostenible de la biomasa energética queda patente en los criterios de sostenibilidad de los apartados 6 y 7 del artículo 29, que establecen una serie de condicionantes que atañen al país de origen de dicha biomasa. El primero requiere que en el Estado donde se recolecte el recurso forestal existan normas de ámbito nacional o subnacional aplicables al aprovechamiento que permitan garantizar la legalidad de las operaciones, la regeneración forestal, la protección de las zonas que se designen legalmente y que la explotación permite mantener o mejorar la capacidad de producción a largo plazo del aprovechamiento. Si no se pudieron verificar estos extremos, la Directiva permite el uso de la biomasa en tanto existan sistemas de gestión a nivel forestal en la zona del aprovisionamiento que garanticen estos mismos requisitos. De este modo, la productividad sostenible puede verse incentivada mediante instrumentos voluntarios, como la certificación de la gestión forestal sostenible, especialmente en situaciones de regulación deficiente.

\subsection{La biomasa forestal circular y los biocarburantes avanzados}

Al hablar de biomasa, no podemos olvidar la posibilidad de aprovechar aquella de origen residual, que además suele resultar más sostenible. El propio sistema de gobernanza prevé en la letra d) del artículo 35 del Reglamento 2018/1999, la elaboración de un informe sobre la sostenibilidad de la bioenergía de la Unión con carácter bienal a partir de 2023. Dicho informe debe ajustarse a las especificaciones del Anexo X.

En concreto, contendrá información sobre los siguientes extremos:

“a) los beneficios y costes medioambientales relativos de los diferentes biocombustibles, biolíquidos y combustibles de biomasa, los efectos de las políticas de importación de la Unión al respecto, las implicaciones en materia de seguridad del abastecimiento y las vías para conseguir un enfoque equilibrado entre producción nacional e importaciones". Precisamente, la aplicación reforzada del principio de proximidad de origen del recurso podría paliar ciertos costes ambientales asociados a la producción de biocombustibles y su importación. Si el problema principal del uso de esta fuente energética son las emisiones inherentes a su combustión, resulta conveniente aplicar políticas tendentes a reducir las emisiones relativas a su ciclo productivo. Por ejemplo, la proximidad del recurso de la instalación donde se emplee permite reducir las asociadas al transporte.

“b) las repercusiones de la producción y la utilización de biomasa sobre la

rural/temas/politica-forestal/efe_2_new_tcm30-155839.pdf

17 BLASCO HEDO, E., Propiedad Forestal Privada y Energías Renovables, Ed. 1a , Thomson - Reuters Aranzadi, 2015. p. 143. 
sostenibilidad en la Unión y en terceros países, incluidas las repercusiones sobre la biodiversidad". Muy conectado al principio de proximidad, la gestión sostenible de los recursos autóctonos, realizada bajo criterios técnicos y sometida a vigilancia y control, podría permitir conjugar el papel protector para la biodiversidad de los espacios agro-silvícolas con una mejor productividad y facilitaría una menor dependencia de la biomasa procedente de terceros países.

“c) datos y análisis de la disponibilidad y la demanda actual y prevista de biomasa sostenible, incluida la repercusión de una mayor demanda de biomasa en los sectores que la utilizan". A este respecto, si bien la biomasa es una fuente marginal para la producción de electricidad, es muy relevante para los sectores del transporte y de la calefacción. A pesar de que en el sector automovilístico se pretende combinar el uso de biocombustibles con la electrificación, parece que aquellos son la mejor solución en el medio plazo para el transporte aéreo y marítimo. Del mismo modo, el impulso de las redes de calefacción urbanas supondrá, previsiblemente, un aumento de la demanda de biocombustibles.

“d) el desarrollo tecnológico y el despliegue de biocombustibles producidos a partir de las materias primas enumeradas en el Anexo IX de la Directiva 2018/2001, y una evaluación de la disponibilidad de materias primas y la competencia por los recursos teniendo en cuenta los principios de la economía circular y la jerarquía de residuos establecidos en la Directiva 2008/98/CE". Esta cuestión se desarrolla en el apartado sobre los biocarburantes avanzados.

“e) información y análisis de los resultados disponibles de investigaciones científicas en materia de cambio indirecto de uso de la tierra en relación con todos los procesos de producción, junto con una evaluación de la posibilidad de reducir el intervalo de incertidumbre detectado en los análisis en que se basan las estimaciones de las emisiones asociadas al cambio indirecto de uso de la tierra y de tener en cuenta el posible impacto de las políticas de la Unión, como la política de medio ambiente, la política en materia de cambio climático o la política agrícola". La incertidumbre acerca de los cambios en los usos indirectos de la tierra y la falta de una metodología fiable para determinar su impacto son interrogantes de máxima importancia para determinar la sostenibilidad de los distintos tipos de biomasa.

“f) respecto tanto de terceros países como de Estados miembros que son una fuente significativa de biocombustibles, biolíquidos y combustibles de biomasa consumidos dentro de la Unión, sobre las medidas nacionales adoptadas para cumplir los criterios de sostenibilidad y ahorro de emisiones de GEI establecidos en el artículo 29, apartados 2 a 7 y 10, de la Directiva 2018/2001, en cuanto a la protección del suelo, el agua y el aire". Dado que el cumplimiento de los criterios de sostenibilidad y de reducción de gases de efecto invernadero se puede articular en torno a medidas de distinta índole, es preciso conocer las acciones concretas que se realizan en cada Estado para evaluar correctamente la sostenibilidad de los distintos tipos de biomasa.

“g) información agregada procedente de la base de datos a que se refiere el artículo 28, apartado 2, de la Directiva 2018/2001", sobre la contabilización de los biocombustibles en el sector del transporte, a efectos de que los proveedores cumplan con sus obligaciones de suministro ${ }^{18}$.

La obtención de materias primas para la producción de biocombustibles es otra de las grandes preocupaciones. En un planeta con una población creciente, es seguro que aumentará la demanda de alimentos y de energía. Así, la cadena de valor de los biocombustibles permite una mayor circularidad de los productos de base biológica, máxime cuando se aplican criterios técnicos para el aprovechamiento de los residuos en los que devienen. En el ámbito científico, a

\footnotetext{
18 ROSA MORENO, J., "El nuevo marco común de la energía renovable”, en VALENCIA MARTÍN, G. y ROSA MORENO, J., La transformación renovable del modelo energético. Thomson Reuters - Aranzadi, 2020, pp. 31-96.
} 
los biocombustibles producidos a partir de materias que no compiten con la producción de alimentos, siendo muchas de éstas de origen residual, se les denomina avanzados. He aquí una particularidad del marco regulatorio de los biocombustibles: la DFERII habla, únicamente, de biocarburantes avanzados, que son aquellos "producidos a partir de las materias primas enumeradas en el Anexo IX parte A" ${ }^{\prime 1}$. De ahí que se considere su menor impacto ambiental y se premie su uso a efectos de su cómputo en la cuota de integración de fuentes de energía renovables mediante la introducción de un multiplicador (x2).

Los biocarburantes avanzados son fundamentales para la descarbonización de determinados sectores considerados críticos, como pueden ser la aviación o el transporte marítimo, o vehículos públicos, que pueden necesitar de una adaptación a medio plazo a través del uso de biocombustibles, pues es difícil que las tecnologías futuras tengan un corto horizonte de implantación generalizada. Por poner el ejemplo del hidrógeno, actualmente la gran mayoría de la producción industrial (en torno al 96\%) procede de combustibles fósiles (lo que se conoce como hidrógeno gris), que generan emisiones de gases de efecto invernadero ${ }^{20}$. Asimismo, actualmente en España sólo hay 8 hidrogeneras operativas (puntos de suministro del gas) ${ }^{21}$, por lo que es lógico que el parque de vehículos sea mínimo. Pese a que se pretende dar un impulso a la generación de hidrógeno procedente de energías renovables, denominado hidrógeno verde, el desarrollo, implantación y generalización de esta tecnología requiere de una gran inversión, por lo que no se prevé que sea viable a corto plazo.

Sin embargo, la calificación como avanzado de los biocarburantes debería ser extensible a los biocombustibles sólidos de base biológica residual. La DFERII vincula dicha calificación, exclusivamente, a los biocarburantes. Dado que un biocarburante es, a tenor del artículo 2.33 de la DFERII, un combustible de biomasa en estado líquido empleado con fines de transporte, se dificulta integrar en este término a los biocombustibles en estado sólido para fines de calefacción, como puedan ser pellets o briquetas que contengan biomasa procedente de residuo silvícola ${ }^{22}$. En el marco de la información sobre la sostenibilidad de la bioenergía, del artículo 35 del Reglamento 2018/1999, que remite a su Anexo X, el texto literal de su letra d), reproducida anteriormente, invita a pensar en una futura extensión de la calificación de "avanzados" a todos los biocombustibles que se produzcan a partir de las materias primas enumeradas en el Anexo IX de la Directiva 2018/2001.

En el plano práctico, la eventual designación de un biocombustible sólido como avanzado, si sus características lo permiten, podría acarrear un doble beneficio. De una parte, convertirlos en merecedores del multiplicador reconocido para los biocarburantes. De otra, estimular el uso de la fracción residual de la biomasa en detrimento de las materias primas brutas. Consecuentemente, estos productos resultarían más atractivos de cara al despliegue de nuevas instalaciones que consumen energía de biomasa, como las redes de calefacción urbana. Y el aumento de la demanda de biocombustibles propiciará, cerrando el círculo, una recuperación de áreas marginales o degradadas para la producción de biomasa, con el consiguiente aumento de la biodiversidad y de la capacidad de absorción de $\mathrm{CO}_{2}$.

\footnotetext{
${ }^{19}$ Artículo 34.2 DFERII.

${ }^{20}$ GONZÁLEZ GARCÍA-CONDE, A., "Introducción a la tecnología del hidrógeno”, en SÁNCHEZ HERVÁS, J. M. et al., Hacia una economía del hidrógeno en España, CIEMAT, Madrid, 2019, pp. 13-33.

${ }^{21}$ Datos actualizados disponibles en https://www.glpautogas.info/hidrogeneras-gasolineras-hidrogeno.html.

${ }^{22}$ Se trata de una de las materias contenidas en el Anexo IX de la DFERII, parte A. Ver letra o).
} 


\subsection{La importancia de las metodologías de contabilización de GEI y de los sistemas de modelización}

Los criterios de sostenibilidad de la DFERII son de aplicación conjunta con los criterios de reducción de gases de efecto invernadero de los biocombustibles.

Efectivamente, la combustión de biomasa supone la vuelta del $\mathrm{CO}_{2}$ capturado a la atmósfera. Sin embargo, existen estudios que demuestran cómo la gestión forestal sostenible puede mejorar la capacidad de absorción de los aprovechamientos silvícolas ${ }^{23}$. Para garantizar que la explotación de un monte no merma su capacidad como sumidero, es necesario aplicar metodologías de contabilización de las emisiones. Si bien el estado actual de la tecnología proporciona herramientas adecuadas a este fin, las mismas pueden acarrear consecuencias adversas.

Una cuestión controvertida de las metodologías de contabilización de emisiones de gases de efecto invernadero es que la gestión forestal se ve limitada por el "nivel de referencia forestal", elemento técnico también controvertido en tanto los Estados con más aprovechamientos forestales podrían llegar a justificar una menor reducción de sus emisiones debido a su capacidad de absorción, por aplicación de los mecanismos de flexibilidad contenidos en el Reglamento UTCUTS ${ }^{24}$.

El uso sostenible de la biomasa con fines energéticos está muy vinculado a los usos de la tierra y a los cambios de uso de la tierra y silvicultura. De modo que se establecen una serie de requisitos que debe cumplir el Estado productor de la biomasa, como ser parte del Acuerdo de París, haber presentado su contribución nacional y disponer de normativa nacional y subnacional aplicable al aprovechamiento de cara a su conservación, refuerzo de las reservas y los sumideros de dióxido de carbono.

En la misma línea, debe acreditarse que las emisiones UTCUTS no superan las absorciones, a la luz del precitado artículo 5 del Acuerdo de París. La contabilización como "cero" de las emisiones procedentes de la combustión de biomasa en el sector energético es una consecuencia de las directrices desarrolladas por el Intergovernmental Panel on Climate Change (IPCC en lo sucesivo), que permite esta fórmula en tanto dichas emisiones se contabilicen en el sector UTCUTS. El Reglamento UTCUTS es una norma de aplicación directa en los Estados miembros y contiene las normas de contabilización de emisiones y absorciones para este sector, así como los correspondientes criterios de comprobación de su cumplimiento. Como se deduce, para cerciorar que el uso energético de la biomasa es sostenible es necesario aplicar metodologías que se superponen. Ello, sin entrar a analizar las especificidades de la contabilidad de las emisiones asociadas a los cambios en los usos indirectos del suelo (CUIT), que asimismo forman parte del complejo entramado de metodologías para determinar cómo se equilibran las emisiones y absorciones de $\mathrm{CO}_{2}$ en la nueva gobernanza climática.

\footnotetext{
${ }^{23}$ RODRIGUEZ-CHÁVES MIMBRERO, B., "Montes y medio ambiente: perspectivas nuevas, nuevos retos", en LOPEZ RAMÓN, F., Observatorio de Políticas Ambientales. Ed.1ª, Aranzadi, CIEDA - CIEMAT, Madrid, 2016, pp. 659-699.

${ }^{24}$ La planificación ambiental de los Estados miembros de la Unión Europea para el periodo 2021 - 2030 prevé obligaciones de reducción de emisiones de $\mathrm{CO}_{2}$ para los sectores previstos en el Reglamento 2018/842 del Parlamento Europeo y del Consejo, de 30 de mayo de 2018, sobre reducciones anuales vinculantes de las emisiones de gases de efecto invernadero por parte de los Estados miembros entre 2021 y 2030 que contribuyan a la acción por el clima, con objeto de cumplir los compromisos contraídos en el marco del Acuerdo de París. Este Reglamento, conocido como de Reparto de Esfuerzos, asigna a los Estados miembros cuotas de reducción de sus emisiones de GEI. A España le asigna una cuota de reducción del 26\% respecto a los niveles de 2005 en su Anexo I. En caso de no alcanzar los objetivos previstos, se facilita que los Estados miembros computen el $\mathrm{CO}_{2}$ atmosférico absorbido por el sector UTCUTS.
} 
Desde luego, la sostenibilidad del uso energético de la biomasa es una cuestión muy compleja. Las distintas directivas de fomento de las energías renovables han sido modificadas, concretándose paulatinamente unos mejores parámetros para esta fuente. Su nicho como fuente energética renovable y neutra en carbono debe establecerse siempre bajo la premisa de demostrar que los bosques de procedencia sean gestionados de manera sostenible. Y en este mismo punto es donde existe un gran margen de mejora, dado que hay una gran superficie boscosa que se encuentra sin gestionar, con las consecuencias climáticas y económicas que eso supone: pérdida de población y empobrecimiento en áreas rurales, mayor incidencia de grandes incendios forestales, degradación del suelo y sequía ${ }^{25}$. La interrelación de las metodologías de contabilización vinculadas a la sostenibilidad del uso energético de la biomasa exige la máxima exactitud y para ello es preciso contar con datos fiables impregnados del elemento de transparencia, cuya presencia en las políticas climáticas puede ser, en cierta medida, cuestionable. Sin embargo, los planes nacionales integrados en materia de energía y clima, como herramienta, pueden aportar algo de luz, pues son una guía para la actividad política durante el próximo decenio.

No obstante, expertos del sector energético recomiendan evaluar la integración de las distintas metodologías de contabilidad con precaución y cuestionan la "neutralización" de las emisiones de sector energético a la luz de la normativa vigente ${ }^{26}$. La conjugación de las distintas metodologías de contabilidad de emisiones disponibles a la hora de evaluar: i) las emisiones procedentes de la combustión de la biomasa; ii) la función como sumidero de carbono que proporciona dicha biomasa; iii) los cambios directos en los usos del suelo; y, iv) los cambios indirectos en los referidos usos (para los que no existen herramientas de contabilidad disponibles ${ }^{27}$ ); es una tarea ardua. Estas metodologías se van perfeccionando con el tiempo a medida que se recaban más datos y se dispone de mejores modelos de predicción y simulación. Un buen ejemplo es el proyecto LIFE FOREST CO $2^{28}$, entre cuyos objetivos se encuentra la modelización de la tasa de secuestro de carbono derivada de actuaciones silvícolas de gestión ${ }^{29}$.

El debate sobre la sostenibilidad de la biomasa es ciertamente necesario, pues determinados aspectos de su aprovechamiento son foco de conflicto entre los distintos grupos de interés, y han servido como punto de partida para el desarrollo de metodologías, técnicas de contabilización de emisiones, estudio de las técnicas de procesado, etcétera. Sin embargo, otras fuentes de generación, como son la energía solar o la eólica, pese a no ser emisivas en la producción energética en sí, tienen un gran impacto en el medio ambiente. Por poner un ejemplo, el desmantelamiento tras el fin de la vida útil de las grandes centrales de producción renovable ha puesto el foco en el problema del tratamiento de residuos y el coste energético de la producción de los dispositivos.

El análisis de ciclo de vida (ACV) resulta una herramienta de utilidad a la hora de

\footnotetext{
${ }^{25}$ RODRIGUEZ-CHAVES MIMBRERO, B., "Montes: la aprobación del Reglamento UTCUTS y el necesario impulso de la gestión forestal”, en LOPEZ RAMÓN, F., Observatorio de Políticas Ambientales. Aranzadi - CIEDA CIEMAT, Madrid, 2019, pp. 838 -873.

${ }^{26}$ RICO, J., "Biomasa forestal para energía: FAO y forestales, sí; ecologistas, no”, Energías Renovables, 2017.

27 COMISIÓN EUROPEA, Informe sobre el estado de la expansión de la producción de cultivos alimentarios y forrajeros pertinentes en todo el mundo, p. 5 [COM (2019) 142 final].

${ }^{28}$ MARTÍNEZ, R., JORDÁN, E., VELAMAZÁN, M. y MARTÍNEZ-FERNÁNDEZ, F., "Proyecto LIFE FOREST $\mathrm{CO} 2$; una propuesta desde la Gestión Forestal Sostenible para una economía baja en carbono", Gestión de montes: servicios ambientales y bioeconomía, $7^{\circ}$ Congreso Forestal Español, 2017.

29 BLASCO HEDO, E., "La gestión de la biomasa garantiza una silvicultura climáticamente inteligente" en REVUELTA PÉREZ. I. y ALONSO MÁS, M. J., La Regulación de la energía limpia ante la emergencia climática. Thomson - Reuters Aranzadi. Cizur Menor, 2020, pp. 387-430.
} 
cuantificar los impactos de cualquier actividad. En los últimos tiempos se han publicado numerosos estudios que, basándose en el empleo de esta metodología, analizan el impacto de las distintas formas de generación de energía ${ }^{30}$. Pero al tratarse de una herramienta fácilmente "configurable", sus resultados pueden ser, en gran medida, manipulables. De esta manera, existen publicaciones que concluyen que, entre los problemas para aplicar el ACV a las distintas fuentes de energía renovable, se encuentran: la amplia diversidad de ubicaciones y contextos que conducen a una variedad de resultados potencialmente no comparable; la existencia de lagunas de datos, especialmente en países donde no existen inventarios o esta práctica de análisis está poco extendida, así como en la extracción de materiales críticos, los cuales son escasos y su disponibilidad a medio plazo puede verse afectada; la transparencia en la metodología y los resultados; la falta de integración en los simuladores de procesos y la falta de consideración de todas las categorías de impacto ${ }^{31}$.

Por lo tanto, es necesario que se establezcan unas bases de análisis en determinadas disciplinas, o de requisitos en los estudios, que permitan establecer comparaciones de calidad, para poder trasladar las mejores opciones a las políticas energéticas. En la misma línea, la interrelación de estrategias tecnológicas para hacer más sostenible la producción de energía renovable, entre ellas, la mejora de los materiales y la eficiencia energética, la reducción de la entrada de energía en la fabricación de componentes, la innovación en el almacenamiento energético intermitente, y el desarrollo de sistemas híbridos; unido a su vez a la implementación de enfoques de economía circular que faciliten el reciclaje y la recuperación de elementos al final de su vida útil, evidencian la necesidad de estudiar cada proyecto de manera individualizada. Como conclusión, las políticas energéticas no pueden fomentar el uso de unas fuentes de energía sobre otras, puesto que su implantación en determinadas zonas puede no ser la opción más adecuada, atendiendo a unas categorías de impacto concretas.

\section{RETICENCIAS AL USO ENERGÉTICO DE LA BIOMASA FORESTAL RESIDUAL}

Centrándonos en el PNIEC español, recientemente evaluado por la Comisión Europea ${ }^{32}$, la Plataforma Ciudadana para una Transición Ecológica Justa presentó alegaciones ${ }^{33}$ al Estudio Ambiental Estratégico ${ }^{34}$ contrarias al uso energético de la biomasa por entender que esta fuente no es sostenible y que su mejor aprovechamiento es su destino al compostaje. Su posición frente a la medida 1.11 - Programas específicos para el aprovechamiento de la biomasa, es contraria a la valorización energética de este residuo por vulnerar la jerarquía de residuos. En este punto,

30 GIBON, T., ARVERSEN, A. y HERTWICH, E. G., "Life cycle assessment demonstrates environmental cobenefits and trade-offs of low-carbon electricity supply options", Renewable and Sustainable Energy Reviews, núm. 76, 2017, pp. 1283-1290; D’AMATO, D., GAIO, M. y SEMENZIN, E., “A review of LCA assessments of forestbased bioeconomy products and processes under an ecosystem services perspective", Science of The Total Environment, núm. 706, 2020; LOZANO MIRALLES, J. A., LÓPEZ GARCÍA, R., PALOMAR CARNICARO, J. M. y REY MARTÍNEZ, F. J., "Comparative study of heat pump system and biomass boiler system to a tertiary building using the Life Cycle Assessment (LCA)”, Renewable Energy, núm. 152, 2020, pp. 1439-1450.

${ }^{31}$ MARTINEZ-HERNÁNDEZ, E. y SIEW NG, K., "Life Cycle Analysis of Renewable Energy", Reference Module in Earth Systems and Environmental Sciences, 2020. https://doi.org/10.1016/B978-0-12-819727-1.00016-9

32 COMISIÓN EUROPEA. Staff Working Document "Assessment of the final national energy and climate plan of Spain" [SWD (2020) 908 final].

33 Disponibles en la página web de la Plataforma para una Transición Ecológica Justa $<$ https://transicionecologicajusta.org/wp-content/uploads/2020/05/OBSERVACIONES-AL-EAE-DEL-PNIECPlataforma-EcoJusta-20200506.pdf $>$.

34 Disponible en la página web del Ministerio para la Transición Ecológica y el Reto Demográfico $<$ https://www.miteco.gob.es/images/es/eae pniec tcm30-506493.pdf>. 
debemos recordar las distintas tipologías de biomasa y cómo la normativa marco europea de residuos, la Directiva 2008/98/CE del Parlamento Europeo y del Consejo, de 19 de noviembre de 2008, sobre los residuos y por la que se derogan determinadas Directivas ${ }^{35}$, excluye explícitamente de su ámbito de aplicación el flujo residual de material natural silvícola destinado a la producción de energía a base de esta biomasa ${ }^{36}$. De modo que la jerarquía de residuos, si bien es aplicable a otros flujos de biorresiduos, no lo es a la biomasa silvícola, incluida la residual, para usos energéticos. De hecho, esta última está bonificada por un doble multiplicador a efectos de su cómputo en la cuota de energía renovable, en virtud de la DFERII $^{37}$.

En materia de economía circular, la Unión Europea lanzó en el año 2018 un paquete legislativo. Sin embargo, la normativa publicada se centró en la regulación de residuos de origen eminentemente abiótico, si bien con importantes precisiones en materia de biorresiduos. Los residuos de biomasa destacan por su heterogeneidad. Entre las distintas fuentes, la silvícola y la procedente de industrias conexas a ese sector generan una importante cantidad de material susceptible de valorización energética. Ello contribuye, asimismo, a mejorar determinados servicios como la prevención de incendios.

Frente a este modelo de gestión de biorresiduos, que permite en última instancia su valorización energética, los residuos procedentes de las placas solares y de los aerogeneradores son de difícil gestión. Los minerales contenidos en este tipo de instalaciones son limitados y desde el sector técnico se baraja la hipótesis de que no contemos con suficientes materiales para el despliegue de la infraestructura que requiere la transición energética. La gestión forestal sostenible ofrece la posibilidad de mejorar la capacidad productiva de los aprovechamientos, pero también de su material vegetal residual.

Por otro lado, las observaciones que realiza la misma Plataforma sobre los objetivos de este PNIEC incluyen el fomento de un modelo distribuido basado en la aproximación de la generación de energía a los centros de consumo, abaratando su precio; el impulso de proyectos colaborativos y el fomento de la economía circular: Dichas observaciones son los pilares del uso de la biomasa forestal procedente de una gestión sostenible de los bosques. EL PNIEC incluye, dentro de los mecanismos de actuación de la citada medida 1.11., el fomento del principio de proximidad y los equipos de calefacción local de alta eficiencia y bajas emisiones, fundamentales para la sustitución de equipos basados en el uso de combustibles fósiles.

\section{EL AUTOCONSUMO COMO ELEMENTO PIVOTE DE LA GENERACIÓN DISTRIBUIDA EN EL NUEVO MODELO A BASE DE ENERGÍAS RENOVABLES}

Tanto la DFERII como el Reglamento 2018/1999 cristalizan la pretensión comunitaria de democratizar la producción de energía a través de las figuras del autoconsumo y del prosumidor $^{38}$. Consecuentemente, los consumidores que busquen autoabastecerse y aquellos que

\footnotetext{
${ }^{35}$ Directiva 2008/98/CE del Parlamento Europeo y del Consejo, de 19 de noviembre de 2008, sobre los residuos y por la que se derogan determinadas Directivas [DO L 312 de 22.11.2008, p. 3/30].

${ }^{36}$ Artículo 2.1.f) de la Directiva 2008/98.

${ }^{37}$ Anexo IX, Parte a), letra o).

38 Sobre la generación distribuida en el ámbito comunitario, se recomienda GALERA RODRIGO, S., "Energía: política energética europea, del cambio de modelo a la mera descarbonización", en LÓPEZ RAMÓN, F., Observatorio de Políticas Ambientales, CIEDA - CIEMAT, Madrid, 2016, pp. 701-721. De la misma autora, "Transición energética en España: retos jurídicos más allá del mercado", en GALÁN VIOQUE, R., GONZÁLEZ RÍOS, I. y LÓPEZ RAMÓN, F., Derecho de las energías renovables y la eficiencia energética en el Horizonte 2020, Ed.1 ${ }^{\text {a }}$, Thomson Reuters Aranzadi, 2017, pp. 499 y ss.
} 
pretendan verter su excedente a la red deberán optar entre las distintas fuentes renovables para satisfacer sus necesidades de suministro. En este contexto, este trabajo pretende arrojar algo de luz acerca de la sostenibilidad de la biomasa frente a otras fuentes, como la fotovoltaica o la mini eólica, máxime cuando a nivel nacional afrontamos el fin de la vida útil de las primeras centrales de generación de energía renovable, que se está demostrando poco sostenible. La dificultad para gestionar los residuos de los aerogeneradores justifica la necesidad de analizar el ciclo de vida de las distintas instalaciones con fines de autoconsumo para poder tildar a esta de limpia y sostenible ${ }^{39}$. Es en el ACV donde se deberían considerar las emisiones de GEI asociadas al ciclo productivo de energía.

El cambio de paradigma en el modelo energético y la transición ecológica, unidas a la democratización y la soberanía energéticas en aras a una mayor participación de todos los sectores sociales, requiere no sólo de políticas de cuantificación de emisiones. La diseminación de los centros de producción, la aparición de la figura del prosumidor y la electrificación del sector del transporte requieren de la adaptación de todo el sistema energético, y pone el foco en distintos aspectos. El PNIEC reconoce, dentro de la dimensión de la descarbonización, la necesidad de adoptar medidas con relación a la gestión de la demanda, almacenamiento y flexibilidad de respuesta de la red (medida 1.2), la adaptación de las redes eléctricas para la integración de renovables (medida 1.3), con el desarrollo del autoconsumo con renovables y generación distribuida (medida 1.4), o de un marco para las energías renovables térmicas (medida 1.6); y establece una relación de políticas en la dimensión de la eficiencia energética. Sin embargo, dicho reconocimiento no deja de ser una descripción somera y nada concreta en términos de metas y políticas a implementar, así como de los escenarios objetivo y tendencial, lo cual no se traduce en proyectos con una inversión determinada y unos mecanismos de evaluación dimensionados dado que, en la práctica, no dejarían de ser implantaciones transversales y sinérgicas de todas las medidas a la vez.

\section{RECAPITULACIÓN Y CONCLUSIONES}

La DFERII prevé unos criterios de sostenibilidad de los combustibles de biomasa referidos al origen o a determinadas instalaciones, cuya aplicabilidad presenta deficiencias que pueden lastrar el uso de los biocombustibles sólidos en el futuro marco de producción energética de Europa. En el caso concreto de la biomasa forestal de origen residual, su uso energético se puede considerar sostenible y más limpio en comparación con otras fuentes renovables, como la fotovoltaica o la eólica, siempre que dicho uso quede condicionado a las prerrogativas ambientales mencionadas en este trabajo. Prerrogativas recogidas por el derecho comunitario y cuya aplicación se concreta a través de parámetros científico-técnicos que, no obstante, todavía tienen margen de mejora.

La implantación de las medidas previstas en el PNIEC requiere de políticas que fomenten determinadas formas de producción y adaptación de las redes en aras a una descentralización de la generación energética y un acercamiento de esta a los puntos de consumo, lo cual invita al futuro prosumidor al autoabastecimiento y al control soberano de su gasto energético, incentivando la concienciación social y una mayor efectividad en la gestión de la demanda doméstica. Sin embargo, todavía queda por explotar, en mayor medida, el potencial de la biomasa como fuente renovable. La planificación energética se centra en el impulso de

\footnotetext{
39 JENSEN, J.P., SKELTON, K., "Wind turbine blade recycling: Experiences, challenges and possibilities in a circular economy", Renewable and Sustainable Energy Reviews, núm. 97, 2018, pp. 165-176.
}

MARSH, G., “What's to be done with 'spent' wind turbine blades?”, Energy Focus, núm. 22-23, 2017, pp. 20-23. 
centrales de energía solar y eólica, infravalorando en cierta medida las posibilidades de diversificación en el mix que ofrece la biomasa como fuente energética flexible y gestionable. Si bien la EAE del PNIEC alerta sobre el impacto en el territorio de la infraestructura para la producción de energía eólica y solar, el planteamiento podría reformularse: ¿Existe alguna fuente renovable cuya producción pueda suponer impactos positivos en el territorio? Este interrogante merece una respuesta elaborada y cimentada en la cautela, pero somos optimistas. Si se despliegan los medios adecuados para cumplir con los objetivos del Acuerdo de París y del Reglamento UTCUTS, extendiendo los esquemas de gestión forestal sostenible, podría alcanzarse esa situación óptima, en la que los montes son espacios ricos en biodiversidad, protectores de esta y proveedores de recursos y servicios para el ser humano.

Finalmente, si se pretende un marco que potencie la soberanía de los consumidores, debe garantizarse que los territorios rurales, donde se ubican principalmente los recursos agrosilvícolas y las industrias asociadas, generadoras de residuos forestales, sean receptores de los beneficios de su impulso.

Este escenario no armoniza con los criterios de sostenibilidad anteriormente mencionados, que a su vez precisan de un marco político sólido para la gestión sostenible de los aprovechamientos forestales. Nos encontramos ante "la pescadilla que se muerde la cola".

La legislación referente a los criterios de sostenibilidad es demasiado abstracta, a pesar de que existen criterios técnicos bastante más precisos, sobre todo a la hora de determinar los impactos de las distintas fuentes de energía renovable a lo largo de toda la vida útil de las instalaciones. En el caso de la biomasa se pone el foco en las emisiones de gases de efecto invernadero, subestimando la importancia de parámetros como la proximidad al recurso o la circularidad del proceso energético en sí, que son los puntos ventajosos frente a otras formas de generación consideradas renovables, como la solar o la eólica, mientras en estas últimas no penalizan suficientemente sus impactos en la implantación y desmantelamiento de las plantas de generación $^{40}$. Resulta recomendable el despliegue de instrumentos de diagnóstico en la generación de energía, para que las políticas puedan enfocarse a orientar las distintas formas de generación de una manera ordenada y eficiente, virando nuestros impactos hacia una transición ecológica más eficaz.

\section{REFERENCIAS BIBLIOGRÁFICAS}

- "Ence ayuda a mantener el suministro de energía renovable durante el temporal Filomena", Europapress, 13 de enero de 2021. Disponible en https://www.europapress.es/economia/energia-00341/noticia-ence-ayuda-mantenersuministro-energia-renovable-temporal-filomena-20210113113442.html

- BELTRÁN CASTELlanOS, J. M., "Instalaciones de energías renovables. El reto de la unificación y simplificación de los procedimientos", en VALENCIA MARTÍN, G. y ROSA MORENO, J., La transformación renovable del modelo energético, Thomson Reuters Aranzadi, Cizur Menor, 2020, pp. 383-426.

\footnotetext{
${ }^{40}$ La Ley 21/2013, de 9 de diciembre, de evaluación ambiental [BOE-A-2013-12913] encomienda la determinación de estos extremos en el estudio de impacto ambiental, regulado en su artículo 5, a cuyo tenor literal, es el "documento elaborado por el promotor que acompaña al proyecto e identifica, describe, cuantifica y analiza los posibles efectos significativos sobre el medio ambiente derivados o que puedan derivarse del proyecto, así como la vulnerabilidad del proyecto ante riesgos de accidentes graves o de catástrofes, el riesgo de que se produzcan dichos accidentes graves o catástrofes y el obligatorio análisis de los probables efectos adversos significativos en el medio ambiente en caso de ocurrencia. También analiza las diversas alternativas razonables, técnica y ambientalmente viables, y determina las medidas necesarias para prevenir, corregir y, en su caso, compensar, los efectos adversos sobre el medio ambiente".
} 
- BLASCO HEDO, E., "La gestión de la biomasa garantiza una silvicultura climáticamente inteligente", en REVUELTA PÉREZ. I. y ALONSO MÁS, M. J., La Regulación de la energía limpia ante la emergencia climática, Ed.1 ${ }^{\mathrm{a}}$, Thomson - Reuters Aranzadi, Cizur Menor, 2020, pp. 387-430.

- BLASCO HEDO, E., Propiedad Forestal Privada y Energías Renovables. Ed. 1a, Thomson - Reuters Aranzadi, Cizur Menor, 2015.

- CAMia, A., GIUNTOl, J., JONSSON, K., ROBERT, N., CAZZANIGA, N., JASINEVIČIUS, G., AVITABILE, V., GRASSI, G., BARREDO CANO, J. I. y MUBAREKA, S., The use of woody biomass for energy production in the EU, Publications Office of the European Union, Luxembourg, 2020.

- COMISIÓN EUROPEA, Comunicación "Legislar mejor para obtener mejores resultados Un programa de la UE" [COM (2015) 215 final].

- COMISIÓN EUROPEA, Estrategia de Biodiversidad para 2030 [COM (2020) 380].

- COMISIÓN EUROPEA, Informe sobre el estado de la expansión de la producción de cultivos alimentarios y forrajeros pertinentes en todo el mundo", p. 5 [COM (2019) 142 final].

- COMISIÓN EUROPEA, Staff Working Document "Assessment of the final national energy and climate plan of Spain" [SWD (2020) 908 final].

- D'AMATO, D., GAIO, M. y SEMENZIN, E., "A review of LCA assessments of forestbased bioeconomy products and processes under an ecosystem services perspective", Science of The Total Environment, núm. 706, 2020.

- GALERA RODRIGO, S., "Energía: política energética europea, del cambio de modelo a la mera descarbonización", en LÓPEZ RAMÓN, F., Observatorio de Políticas Ambientales, Ed.1 ${ }^{\text {a }}$, Aranzadi, CIEDA - CIEMAT, Madrid, 2016, pp. 701-721.

- GALERA RODRIGO, S., "Transición energética en España: retos jurídicos más allá del mercado", en GALÁN VIOQUE, R., GONZÁLEZ RÍOS, I. y LÓPEZ RAMÓN, F., Derecho de las energías renovables y la eficiencia energética en el Horizonte 2020, Ed. $1^{\text {a }}$, Thomson Reuters Aranzadi, Cizur Menor, 2017, pp. 499-513.

- GIBON, T., ARVERSEN, A. y HERTWICH, E. G., "Life cycle assessment demonstrates environmental co-benefits and trade-offs of low-carbon electricity supply options" Renewable and Sustainable Energy Reviews, núm. 76, 2017, pp. 1283-1290.

- GONZÁLEZ GARCÍA-CONDE, A., "Introducción a la tecnología del hidrógeno", en SÁNCHEZ HERVÁS, J. M. (et al.), Hacia una economía del hidrógeno en España, CIEMAT, 2019, Madrid, pp. 13-33.

- JENSEN, J. P. y SKELTON, K., "Wind turbine blade recycling: Experiences, challenges and possibilities in a circular economy", Renewable and Sustainable Energy Reviews, núm. 97, 2018, pp. 165-176.

- Ley 21/2013, de 9 de diciembre, de evaluación ambiental.

- LOZANO MiRAlleS, J. A., LÓPEZ GARCÍA, R., PALOMAR CARNiCARO, J. M. y REY MARTÍNEZ, F. J., "Comparative study of heat pump system and biomass boiler system to a tertiary building using the Life Cycle Assessment (LCA)", Renewable Energy, núm. 152, 2020, pp. 1439-1450. 
- MARSH, G., "What's to be done with 'spent' wind turbine blades?", Renewable Energy Focus, núm. 22-23, 2017, pp. 20-23.

- MARTÍNEZ, R., JORDÁN, E., VELAMAZÁN, M. y MARTÍNEZ-FERNÁNDEZ, F., "Proyecto LIFE FOREST CO2; una propuesta desde la Gestión Forestal Sostenible para una economía baja en carbono", Gestión de montes: servicios ambientales y bioeconomía, $7^{\circ}$ Congreso Forestal Español, 2017.

- MARTINEZ-HERNÁNDEZ, E. y SIEW NG, K., "Life Cycle Analysis of Renewable Energy", Reference Module in Earth Systems and Environmental Sciences, 2020.

- MINISTERIO DE AGRICULTURA, PESCA Y ALIMENTACIÓN, Estrategia Forestal Española. Segunda parte; Propuestas de Política Forestal, 22 de diciembre de 2020. Disponible en https://www.mapa.gob.es/es/desarrollo-rural/temas/politicaforestal/efe 2 new tcm30-155839.pdf

- MINISTERIO PARA LA TRANSICIÓN ECOLÓGICA Y EL RETO DEMOGRÁFICO, Plan Nacional Integrado de Clima y Energía 2021 - 2030. Disponible en https://www.miteco.gob.es/images/es/eae_pniec_tcm30-506493.pdf https://www.miteco.gob.es/images/es/pnieccompleto_tcm30-508410.pdf

- MINISTERIO PARA LA TRANSICIÓN ECOLÓGICA Y EL RETO DEMOGRÁFICO. Proyecto de Ley de Cambio Climático y Transición Energética. Disponible en https://www.miteco.gob.es/es/ministerio/proyectodeleydecambioclimaticoytransicionenerge tica_tcm30-509256.pdf

- MOVIMIENTO MUNDIAL POR LOS BOSQUES TROPICALES, "La transformación de centrales a carbón para combustión de biomasa sólo reemplaza un desastre por otro", Boletín Mensual, núm. 189, 2013, 7 de septiembre de 2020. Disponible en https://wrm.org.uy/es/articulos-del-boletin-wrm/seccion2/la-transformacion-de-centrales-acarbon-para-combustion-de-biomasa-solo-reemplaza-un-desastre-por-otro/

- PARLAMENTO EUROPEO, Directiva 2008/98/CE del Parlamento Europeo y del Consejo, de 19 de noviembre de 2008, sobre los residuos y por la que se derogan determinadas Directivas [DO L 312 de 22.11.2008, p. 3/30].

- PARLAMENTO EUROPEO, Directiva 2018/2001, del Parlamento Europeo y del Consejo, de 11 de diciembre de 2018, relativa al fomento del uso de energía procedente de fuentes renovables [DO L 328 de 21.12.2018, p. 82/209].

- PARLAMENTO EUROPEO, Reglamento 2018/1999, del Parlamento Europeo y del Consejo, de 11 de diciembre de 2018, sobre la gobernanza de la Unión de la Energía y de la Acción por el Clima [DO L 328 de 21.12.2018, p. 1/77].

- PLATAFORMA PARA UNA TRANSICIÓN ECOLÓGICA JUSTA, Observaciones a la Evaluación Ambiental Estratégica del Plan Nacional Integrado de Clima y Energía. Disponible en https://transicionecologicajusta.org/wpcontent/uploads/2020/05/OBSERVACIONES-AL-EAE-DEL-PNIEC-Plataforma-EcoJusta20200506.pdf

- Resolución de 30 de diciembre de 2020, de la Dirección General de Calidad y Evaluación Ambiental, por la que se formula la declaración ambiental estratégica del Plan Nacional Integrado de Energía y Clima 2021-2030.

- RICO, J., "Biomasa forestal para energía: FAO y forestales, sí; ecologistas, no", Energías Renovables, 2017. 
- RICO, J., "Claro que podíamos presentar biomasa a la subasta, pero a un precio inasumible", Energías Renovables, 29 de enero de 2021. Disponible en https://www.energias-renovables.com/biomasa/a-claro-que-podiamos-presentar-biomasa-a$\underline{20210129}$

- RICO, J., "La posible modificación de los criterios de sostenibilidad de la bioenergía desata el debate en Europa", Energías Renovables, 21 de enero de 2021. Disponible en https://www.energias-renovables.com/biomasa/la-posible-modificacion-de-los-criterios-de$\underline{20210121}$

- RICO, J., "Un informe de la CE sugiere que los criterios de sostenibilidad se apliquen a todos los productos forestales, no solo a la bioenergía". Energías Renovables, 4 de febrero de 2021. Disponible en https://www.energias-renovables.com/biomasa/un-informe-de-la-cesugiere-que-20210204

- RODRÍGUEZ CHAVES-MIMBRERO, B., "Apuesta de los montes como sumideros de carbono y como fuente de riqueza en el ámbito rural", en LOPEZ RAMÓN, F., Observatorio de Políticas Ambientales, Ed.1 ${ }^{\mathrm{a}}$, Aranzadi, CIEDA - CIEMAT, Madrid, 2017, pp. 455-482.

- RODRÍGUEZ CHAVES-MIMBRERO, B., "Montes y medio ambiente: perspectivas nuevas, nuevos retos”, en LOPEZ RAMÓN, F., Observatorio de Políticas Ambientales. Ed.1 ${ }^{\text {a }}$, Aranzadi, CIEDA - CIEMAT, Madrid, 2016, pp. 659-699.

- RODRÍGUEZ CHAVES-MIMBRERO, B., "Montes: la aprobación del Reglamento UTCUTS y el necesario impulso de la gestión forestal", en LOPEZ RAMÓN, F., Observatorio de Políticas Ambientales, Ed. 1ª, Aranzadi, CIEDA - CIEMAT, Madrid, 2019, pp. 838-873.

- RODRÍGUEZ CHAVES-MIMBRERO, B., "Montes: la gestión sostenible en la normativa de última generación”, en LOPEZ RAMÓN, F., Observatorio de Políticas Ambientales, Ed.1 ${ }^{a}$, Aranzadi, CIEDA - CIEMAT, 2015, Madrid, pp. 645-693.

- ROSA MORENO, J., "El nuevo marco común de la energía renovable", en VALENCIA MARTÍN, G. y ROSA MORENO, J., La transformación renovable del modelo energético, Thomson Reuters - Aranzadi, 2020, pp. 31-96. 